\title{
ChemComm
}

\section{N,S-Dimethyldithiocarbamyl Oxalates as Precursors for Determining Kinetic Parameters for Oxyacyl Radicals}

\begin{tabular}{|r|l|}
\hline Journal: & ChemComm \\
\hline Manuscript ID: & CC-COM-08-2014-006132.R1 \\
\hline Article Type: & Communication \\
\hline Date Submitted by the Author: & 20-Aug-2014 \\
\hline Complete List of Authors: & $\begin{array}{l}\text { Kyne, Sara; Universite Pierre et Marie Currie Paris 6, Institut Parisien de } \\
\text { Chimie Moléculaire } \\
\text { Schiesser, Carl; The University of Melbourne, School of Chemistry }\end{array}$ \\
\hline
\end{tabular}




\title{
N,S-Dimethyldithiocarbamyl Oxalates as Precursors for Determining Kinetic Parameters for Oxyacyl Radicals†
}

\author{
Sara H. Kyne ${ }^{a, b, c}$ and Carl H. Schiesser* ${ }^{a, b}$ \\ Received (in XXX, XXX) Xth XXXXXXXXX 20XX, Accepted Xth XXXXXXXXX 20XX \\ ${ }_{5}$ DOI: 10.1039/b000000x
}

N,S-Dimethyldithiocarbamyl oxalates (eg. 6, 10) are novel, readily prepared precursors to alkyloxyacyl radicals 1 that are more suitable for kinetic studies than existing precursors; 10 has allowed the determination of accurate rate data for the 10 cyclization of the butenyloxyacyl radical $5\left(k_{c}=1.2 \times 10^{7} \mathrm{~s}^{-1}\right.$ at $21^{\circ} \mathrm{C}$ ).

Methods for the generation of carbon-centred free radicals abound. ${ }^{1}$ Halides, arylselenides, aryltellurides, xanthates, pyridinethioneoxycarbonyl (PTOC, Barton) ${ }^{2}$ esters and 15 dithiocarbamyl (Kim) esters, ${ }^{3}$ while most commonly used, form just a subset of the chemical toolbox available to the practitioner when considering the use of radical chemistry in synthesis. ${ }^{1}$

Despite this, there are still some classes of carbon-centred radical for which no robust precursors have been reported. ${ }_{20}$ Oxyacyl radicals (1) are one such class of radical. ${ }^{4}$ To date only phenylselenide (2a), ${ }^{5}$ phenyltelluride $(\mathbf{2 b})^{6}$ and PTOC oxalate $(3)^{7}$ precursors have appeared in the literature (Scheme 1), and these have their own specific limitations and drawbacks. For example, studies using tellurides (2b) and PTOC oxalates (3) are 25 complicated by photochemical instability, ${ }^{7,8}$ while the former (2a) can be contaminated with traces of diphenyl diselenide, ${ }^{9}$ which for the purposes of synthesis can often present no real disadvantage, however for those of us interested in determining accurate rate data, this contaminant provides some significant 30 challenges. Diphenyl diselenide is known to react rapidly with chain-carrying reagents such as tributyltin hydride to afford benzeneselenol (Scheme 1). ${ }^{9}$ This impurity reacts some three orders of magnitide faster as a hydrogen atom donor than the intended stannane, ${ }^{10}$ and even undetectable levels of diphenyl 35 diselenide contamination can skew the interpretation of the kinetic data.

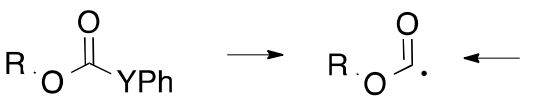

$$
\begin{aligned}
& \text { 2a: } Y=S e \\
& \text { b: } Y=T e \\
& 1 \\
& { }_{3}{ }_{\mathrm{O}^{\mathrm{O}}}^{\mathrm{O}} \\
& \mathrm{PhSeSePh}+\mathrm{Bu}_{3} \mathrm{SnH} \longrightarrow \mathrm{Bu}_{3} \mathrm{SnSePh}+\mathrm{PhSeH} \\
& \left.k_{H} \sim 10^{6} \mathrm{M}^{-1} \mathrm{~s}^{-1} \downarrow \mathrm{R}^{\cdot} \quad k_{H} \sim 10^{9} \mathrm{M}^{-1} \mathrm{~s}^{-1}\right|^{\circ} \\
& \mathrm{RH} \\
& \mathrm{RH}
\end{aligned}
$$

With this in mind, we recently reported the use of high-level computational techniques for the determination of kinetic data for the 5-exo and 6-endo modes of ring closure of a series of 40 substituted acyl and oxyacyl radicals. ${ }^{4}$ While the calculated rate constants $\left(k_{c}\right)$ for the cyclization of the acyl radicals (eg. 4) were in good agreement with available experimental data, we reported that the oxyacyl system (5) cyclizes with a rate constant of $8 \mathrm{x}$ $10^{7} \mathrm{~s}^{-1}\left(80^{\circ} \mathrm{C}\right)$ using G3(MP2)-RAD, well over an order of 45 magnitude faster than that reported by Newcomb $\left(4 \times 10^{6} \mathrm{~s}^{-1}\right){ }^{7}$ based on data provided by Bachi. ${ }^{5}$ We argued that this discrepancy was largely the result of $\mathrm{PhSeSePh}$ contamination during the experiments performed by Bachi, a situation acknowledged by Newcomb when he concluded that "one must 50 be aware of the fact that generation of $\mathrm{PhSeH}$ from small impurities of $\mathrm{PhSeSePh}$ or from photolysis can be problematical". 7

In search of a more robust oxyacyl radical precursor appropriate for kinetic studies, we explored the possibility that ${ }_{55}$ "Kim esters" derived from oxalic acid may be suitable for this purpose. $^{3}$
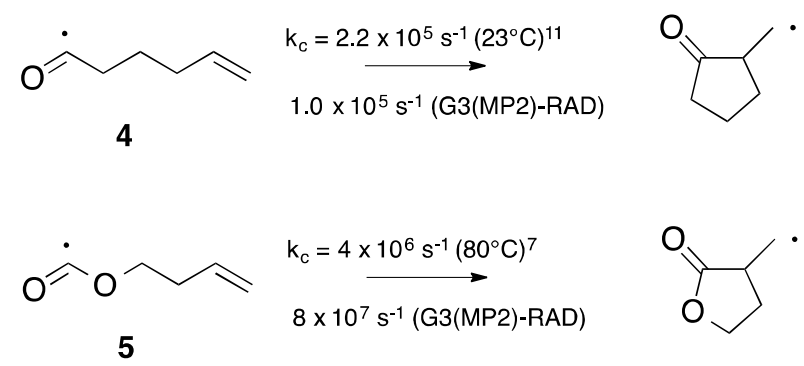

Scheme 2.

We now report that alkyl $N, S$-dimethyldithiocarbamyl oxalate esters (eg. 6) are readily prepared compounds that generate oxyacyl radicals under photochemical conditions. In addition, 60 these novel precursors have allowed us to kinetically "recallibrate" the cyclization of $\mathbf{5}$; the value of $k_{c}$ reported herein is in excellent agreement with our previously reported calculations.

N,S-dimethyldithiocarbamyl esters (Kim esters) were ${ }_{65}$ developed by Kim as more robust precursors for alkyl radicals than the corresponding pyridinethioneoxycarbonyl (PTOC) esters reported by Barton a few decades ago, ${ }^{3}$ and we have successfully employed these precursors on several occasions. ${ }^{12,13}$ Guided by this previous work, 1-octanol was stirred in oxalyl chloride

Scheme 1. 


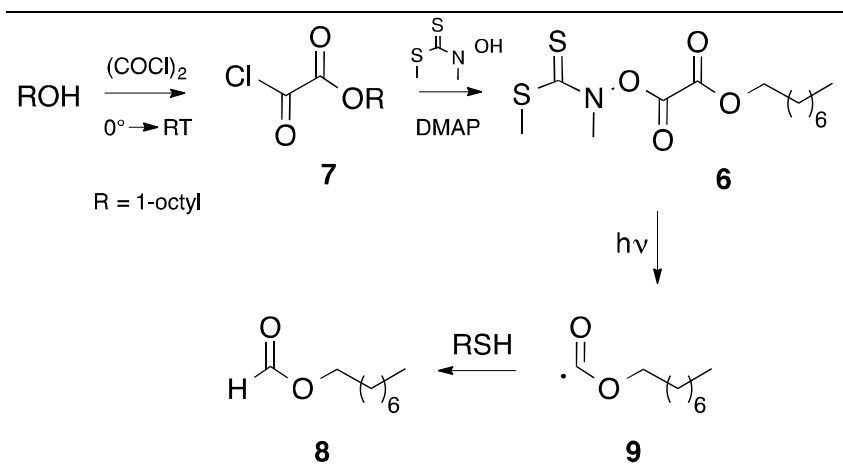

Scheme 3.

overnight. Following removal of the solvent in vacuo, the residue, presumed to be the chloride 7 was dissolved in dichloromethane and further reacted with $N$-hydroxy- $N, S$ dimethyldithiocarbamate ${ }^{3}$ and a cataytic amount of DMAP in 5 dichloromethane to afford 6 in $94 \%$ yield (Scheme 3). The dithiocarbamate $\mathbf{6}$ was not stable to chromatography, however, in our hands, with the correct stoichiometry and careful workup, 6 could be isolated with excellent purity.

When a solution of 6 and tert-dodecanethiol ( $\mathrm{RSH}, 0.6 \mathrm{M})$, in 10 benzene, was irradiated in a Rayonet photochemical reactor ( 350 $\mathrm{nm}$ ) for one hour, we were delighted to observe the quantitative formation of 1-octyl formate (8) by GC analysis and direct comparison with an authentic standard; presumably $\mathbf{8}$ is formed from the intermediate oxyacyl radical 9. This outcome 15 demonstrates the synthetic utility of this novel radical precursor.

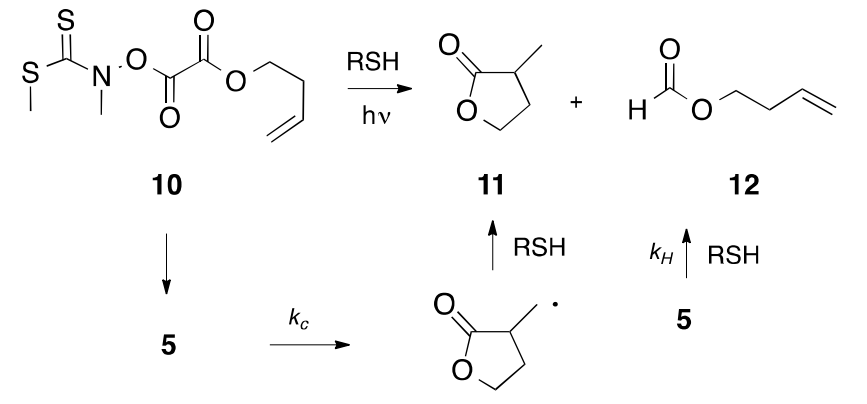

$$
\frac{\mathrm{d}[\mathbf{1 2}]}{\mathrm{d}[\mathbf{1 1}]}=\frac{k_{H}[\mathrm{RSH}]}{k_{c}} \ldots(1) \Rightarrow \frac{[12]}{[11]}=\frac{k_{H}[\mathrm{RSH}]}{k_{c}} \ldots \text { (2) }
$$

Scheme 4.

We next turned our attention to the cyclization of the 3butenyloxyacyl radical 5. The dithiocarbamyl precursor $\mathbf{1 0}$ was prepared in identical manner to $\mathbf{6}$ from 3-butenol and was isolated in $85 \%$ yield. ${ }^{\dagger}$ Given that the rate constant $\left(k_{H}\right)$ for hydrogen 20 transfer from tributyltin hydride to an oxyacyl radical $\mathbf{1}$ has been previously reported, ${ }^{7}$ initial experiments were carried out under photochemical conditions (Rayonet) in benzene using $\mathbf{1 0}$ and $\mathrm{Bu}_{3} \mathrm{SnH}$. Disappointingly, these experiments provided reaction mixtures that were difficult to analyse by GC because of broad 25 overlapping signals that we ascribed to tin-based byproducts. Consequently, an alternative kinetic paradigm was required.

To our delight, when this reaction was repeated using tertdodecanethiol as the source of hydrogen atom, GC analysis of the crude reaction mixture revealed cleanly the formation of 30 methylbutyrolactone $\mathbf{1 1}$ and butenylformate $\mathbf{1 2}$ by comparison
Table 1. Relative rate data $\left(21^{\circ} \mathrm{C}\right)$ for the ring closure of the butenyloxyacyl radical 5 in benzene. Reactions performed under pseudo first-order conditions in tert-dodecanethiol (RSH).

\begin{tabular}{cccc}
\hline $\begin{array}{c}{[\mathrm{RSH}]} \\
(\mathrm{M})\end{array}$ & {$[\mathbf{1 2}] /[\mathbf{1 1}]^{\mathrm{a}}$} & $\begin{array}{c}k_{H} / k_{c} \\
\left(\mathrm{M}^{-1}\right)\end{array}$ & $\begin{array}{c}k_{c}^{\mathrm{b}} \\
\left(\mathrm{s}^{-1}\right)\end{array}$ \\
\hline 0.43 & 0.27 & 0.63 & $1.1 \times 10^{7}$ \\
0.75 & 0.41 & 0.55 & $1.2 \times 10^{7}$ \\
1.20 & 0.64 & 0.53 & $1.3 \times 10^{7}$ \\
2.00 & 1.09 & 0.55 & $1.3 \times 10^{7}$ \\
2.50 & 1.46 & 0.58 & $1.2 \times 10^{7}$ \\
3.00 & 1.76 & 0.59 & $1.2 \times 10^{7}$
\end{tabular}

aAverage of three experiments. ${ }^{b}$ Spot value calculated from equation 2 (see text).

with authentic samples (Scheme 4). When repeated with a tenfold excess of thiol (pseudo first-order conditions) in benzene $\left(21^{\circ} \mathrm{C}\right)$ at the concentrations listed in Table 1 , ratios of $[\mathbf{1 2}] /[\mathbf{1 1}]$ consistent with radical chemistry (Scheme 4) were observed 35 (Table 1). The relative rate constant $k_{H} / k_{c}$ is obtained by linear regressional analysis of the data in Table 1 (Figure 1) and application of the integrated rate equation (eqn. 2).

Inspection of Figure 1 reveals excellent linearity $\left(R^{2}=0.996\right)$ and confirms the validity of equation 2 ; a value of the relative 40 rate constant $\left(k_{H} / k_{c}\right)$ of $0.59 \pm 0.02$ is obtained. Determining a value for $k_{c}$ requires knowledge of the rate constant for hydrogen atom transfer $\left(k_{H}\right)$ from tert-dodecanethiol (RSH); unfortunately this rate constant has not been determined for oxyacyl radicals $\mathbf{1}$.

It is well established that unlike acyl radicals, oxyacyl radicals 45 are not stablised by resonance, as evidenced by IR spectroscopy and computational studies. ${ }^{11,14}$ It is somewhat surprising therefore that the reported value of $k_{H}$ for radicals 1 reacting with $\mathrm{Bu}_{3} \mathrm{SnH}\left(1.1 \times 10^{6} \mathrm{M}^{-1} \mathrm{~s}^{-1}, 80^{\circ} \mathrm{C}\right)^{7}$ is almost identical to that for acyl radicals (eg. 4). ${ }^{15}$ On the basis of relative radical ${ }_{50}$ stabilities, ${ }^{14}$ we would expect 5 to react significantly faster with tert-dodecanethiol than its acyl counterpart (4). CCSD(T)/augcc-pVDZ calculations reported previously suggest that $\mathbf{5}$ is about $45 \mathrm{~kJ} \mathrm{~mol}^{-1}$ less stable than $4 .^{14}$ Applying this same methodology to other radicals reveals that a primary alkyl radical (eg. ethyl) is 55 about $55 \mathrm{~kJ} \mathrm{~mol}^{-1}$ less stable than an acyl radical, while a vinylic radical (eg. ethylenyl) is calculated to be about $98 \mathrm{~kJ} \mathrm{~mol}^{-1}$ less

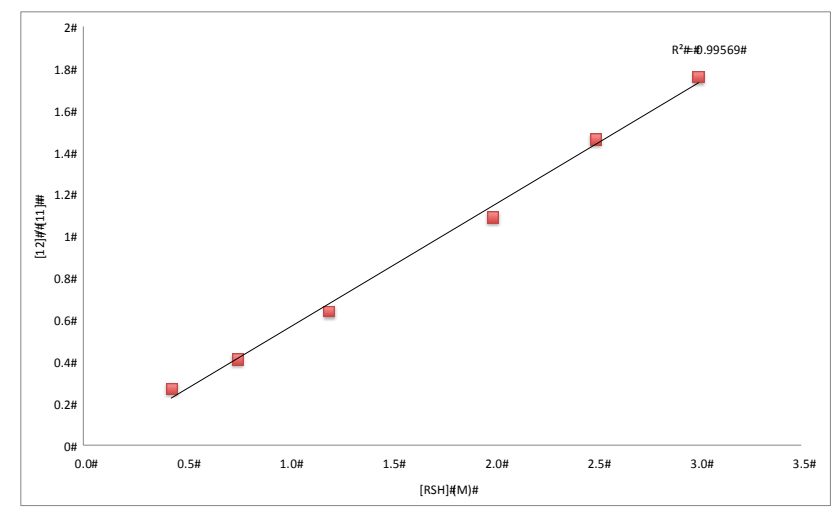

Figure 1. Dependence of $[12] /[11]$ on tert-dodecanethiol concentration at $21^{\circ} \mathrm{C}$ for the cyclization of 5 in benzene. 
stable than 4 at CCSD(T)/aug-cc-pVDZ ${ }^{\S}$ These data, in turn, suggest that an oxyacyl radical such as $\mathbf{5}$ is more similar in reactivity to a primary alkyl radical than an acyl radical. Importantly, $\mathbf{5}$ would not be expected to react with a rate constant 5 of an acyl radical (too slow), or a vinyl radical (too fast).

With this in mind, we suggest that $\mathbf{5}$ abstracts hydrogen atom from tert-dodecanethiol with a similar rate constant $\left(k_{H}\right)$ to that of a primary alkyl radical, namely $6.8 \times 10^{6} \mathrm{M}^{-1} \mathrm{~s}^{-1}$ at $21^{\circ} \mathrm{C} .{ }^{16 \pi}$ With this assumption, our value of $k_{H} / k_{c}$ leads to:

10

$$
k_{c}=1.2 \times 10^{7} \mathrm{~s}^{-1}\left(21^{\circ} \mathrm{C}\right) \text {. }
$$

This value of $k_{c}$ is in excellent agreement with our calculated (G3(MP2)-RAD) value of $1.5 \times 10^{7} \mathrm{~s}^{-1}\left(21^{\circ} \mathrm{C}\right)^{4}$ and significantly 15 faster than the value reported previously. ${ }^{7}$

Not only are we delighted with the agreement between our calculated and experimental data, this study provides further validation of our computational methodology for determining rate data and also suggests that our assumption regarding $k_{H}$ for 20 oxyacyl radicals is approximately correct.

Alternatively, using our calculated value of $k_{c}$, we can determine a value for $k_{H}$ of $7 \times 10^{6} \mathrm{~s}^{-1}$, slighly (but not significantly) higher than the value used earlier based on the experimental value for a primary alkyl radical (vide supra).

25 Finally, we are now in a position to revisit the the work of Bachi and Bosch and provide a more robust value for $k_{H}$ for $\mathrm{Bu}_{3} \mathrm{SnH}$ reduction of $\mathbf{1}$ by the application of equation 2. While

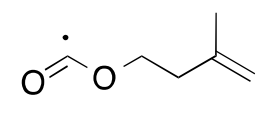

13

the authors report data for the ring-closure of $\mathbf{5}$, product ratios are reported in neat $\mathrm{Bu}_{3} \mathrm{SnH}^{5}$ Consequently, it is difficult to 30 determine reliable reagent concentrations and, from them, to calculate rate constants. On the other hand, more robust data are provided for the cyclization of the related radical 13. Using the reported product ratio and average value of $\left[\mathrm{Bu}_{3} \mathrm{SnH}\right],{ }^{5}$ together with our value of $k_{c}\left(2.7 \times 10^{7} \mathrm{~s}^{-1}, 80^{\circ} \mathrm{C}\right)^{4}$ for 13 , we suggest that $354.6 \times 10^{6} \mathrm{M}^{-1} \mathrm{~s}^{-1}\left(80^{\circ} \mathrm{C}\right)$ is a more appropriate value for $k_{H}$ when tributyltin hydride reacts with oxyacyl radicals 1 . This value for $k_{H}$ is well aligned with that for a primary alkyl radical reacting with $\mathrm{Bu}_{3} \mathrm{SnH}$, namely $6.4 \times 10^{6} \mathrm{M}^{-1} \mathrm{~s}^{-1}\left(80^{\circ} \mathrm{C}\right){ }^{18}$

We thank the Australian Research Council through the Centres 40 of Excellence Scheme for generous financial support.

\section{Notes and references}

${ }^{a} A R C$ Centre of Excellence for Free Radical Chemistry and Biotechnology, Australia

${ }^{b}$ School of Chemistry and Bio21 Molecular Science and Biotechnology 45 Institute, The University of Melbourne, Victoria, 3010, Australia. Fax: +61 39347 8189; Tel: +61 38344 2432; E-mail: carlhs@unimelb.edu.au ${ }^{c}$ Current address: Institut Parisien de Chimie Moléculaire, Université Pierre et Marie Curie, Sorbonne Universités - UMPC Univ. Paris 6, 75005 Paris, France.

50

$\dagger$ Electronic Supplementary Information (ESI): experimantal details for the preparation of $\mathbf{6}$ and 10. General protocol for kinetic experiments. ${ }^{1} \mathrm{H}$

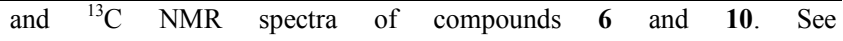
DOI: $10.1039 / \mathrm{b} 000000 \mathrm{x} /$

$55 \S$ This work.

TThis is $k_{H}$ for a primary alkyl radical (5-hexenyl) reacting with tertbutylthiol (ref. 16). While many workers have assumed that tertdodecanethiol reacts with the same rate constant as tert-butylthiol, we have recently verified, in independent work, that the two reagents react 60 with primary alkyl radicals with rate constants $\left(k_{H}\right)$ within experimental error of each other (ref. 17).

1 Encyclopedia of Radicals in Chemistry, Biology and Materials, ed. C. 65 Chatgilialoglu and A. Studer, John Wiley \& Sons Ltd, Chichester, UK, 2012.

2 D. H. R. Barton, D. Crich and W. B. Motherwell, Tetrahedron, 1985, 41, 3901.

3 S. Kim, C. J. Lim, S.-E. Song and H.-Y.. Kang, Chem. Commun., $70 \quad 2001,1410$

4 A. N. Hancock and C. H. Schiesser, Chem. Sci., 2014, 5, 1967.

5 M. D. Bachi and E. Bosch, J. Org. Chem., 1992, 57, 4696.

6 M. A. Lucas and C. H. Schiesser, J. Org. Chem., 1998, 63, 3032.

7 P. A. Simakov, F. N. Martinez, J. H. Horner and M. Newcomb, 1998, $75 \quad 63,1226$.

8 M. A. Lucas and C. H. Schiesser, J. Org. Chem., 1996, 61, 5754.

9 D. Crich and Q. W. Yao, J. Org. Chem., 1995, 60, 84.

10 M. Newcomb, Tetrahedron, 1993, 49, 1151.

11 C. E. Brown, A. G. Neville, D. M. Rayner, K. U. Ingold and J. 80 Lusztyk, Aust. J. Chem., 1995, 48, 363.

12 M. W. Carland, R. L. Martin and C. H. Schiesser, Org. Biomol. Chem., 2004, 2, 2612.

13 T. Fenner, J. M. White and C. H. Schiesser, Org. Biomol. Chem., 2006, 4, 466.

8514 T. Morihovitis, C. H. Schiesser and M. A. Skidmore, J. Chem. Soc. Perkin Trans. 2, 1999, 2041.

15 C. Chatgilialoglu, C. Ferreri, M. Lucarini, P. Pedrielli and G. F. Pedulli, Organometallics, 1995, 36, 1299.

16 M. Newcomb, A. G. Glenn and M. B. Manek, J. Org. Chem., 1989, $90 \quad \mathbf{5 4}, 4603$.

17 A. H. Hancock and C. H. Schiesser, unpublished.

18 L. J. Johnson, J. Lusztyk, D. D. M. Wayner, A. N. Abeywickrema, A. L. J. Beckwith, J. C. Scaiano and K. U. Ingold, J. Am. Chem. Soc., 1985, 107, 4594. 